\title{
Changes in the Th9 cell population and related cytokines in the peripheral blood of infants with recurrent wheezing
}

\author{
GUANGLIANG LIU ${ }^{1, *}$, LEI QIAN ${ }^{2, *}$, TONGHUI XU ${ }^{1}$, JIANXIU YU⿱2, MING LI ${ }^{2}$, YUBAO CUI ${ }^{3, *}$ \\ ${ }^{1}$ Department of Pediatrics, Binhai County People's Hospital, Bianhai, Jiangsu Province, China \\ ${ }^{2}$ Department of Laboratory Medicine, Binhai County People's Hospital, Bianhai, Jiangsu Province, China \\ ${ }^{3}$ Department of Clinical Laboratory, Wuxi People's Hospital Affiliated to Nanjing Medical University, Wuxi, China \\ "These authors contributed equally to this work.
}

\begin{abstract}
Introduction: Thelper type 9 (Th9) cells have been shown to play a key role in initiating allergic reactions and promoting airway inflammation. However, to the best of our knowledge, their role has not been analyzed in infants with recurrent wheezing.

Material and methods: We performed a case-control study including 34 infants with recurrent wheezing and the same number of healthy infants as controls; all subjects were aged 1-to 3-years-old. The Th9 cell populations in the peripheral blood of these subjects were analyzed using flow cytometry, along with the assessment of Th9- and Th2-related plasma cytokine levels, including interleukin (IL)-1 $\beta$, $I L-4, I L-5, I L-9, I L-10, I L-13, I L-17 A$, and IL-33, and transforming growth factor $\beta 1$ (TGF- $\beta 1)$ using a Luminex 200 immunoassay.

Results: Our results indicated that infants with recurrent wheezing had higher percentages of Th9 cells (median, 0.69\%; range, 0.46-1.08\%) as compared to healthy infants (median, $0.25 \%$, range, $0.13-0.36 \% ; p<0.05)$. In addition, infants with recurrent wheezing also exhibited higher plasma levels of cytokines $I L-4, I L-9, I L-10, I L-33$, and TGF- $\beta 1$. Furthermore, the percentage of Th9 cells was positively correlated with the levels of IL-4 $(r=0.408, p<0.05)$ and $I L-9(r=0.644, p<0.05)$ in the peripheral blood of wheezing infants.

Conclusions: Our findings suggest that the percentage of Th9 cells is increased in infants with recurrent wheezing; thus, Th9 cells may play an important role in the pathogenesis of recurrent wheezing.
\end{abstract}

Key words: wheezing, Th9 cells, infants, interleukin 9, interleukin 4.

(Centr Eur J Immunol 2020; 45 (1): 60-68)

\section{Introduction}

Wheezing is the most common respiratory symptom in infants. It is characterized by the narrowing of the airways and hyper-responsiveness, thus resulting in difficult breathing. It has a long-term impact on the airway due to inflammation, and contributes to higher morbidity in infants than for any other age group [1]. Complex interactions between airway microbes, environmental triggers, and innate immune responses play an important role in the pathogenesis of wheezing $[1,2]$. Epidemiological studies have demonstrated that the etiology of recurrent wheezing includes allergies and infections while infants attend daycare, respiratory tract infections, and early wheezing [3]. Specifically, recurrent wheezing has been shown to increase the risk of asthma development at later stages, when more than half of all children show persistent asth- ma symptoms before the age of 3 [4]. Surprisingly, the systematic understanding of recurrent wheezing in infants remains poorly understood.

Th9 cells, which are characterized as the interleukin (IL)-9-CD4+ $\mathrm{T}$ cell subset, are believed to be critical for developing allergic reactions and initiating airway inflammation. In a mouse model, the transfer of antigen-specific Th9 cells has resulted in asthma-associated airway inflammation. In addition, it has been shown that PU.1 and interferon-regulatory factor 4 (IRF4) are the key transcription factors, through which Th9 cells regulate IL-9 production [5]. Atopic infants have the predisposition to develop IL-9-secreting T cells [6]. Children allergic to peanuts also display increased levels of Th9 cells and IL-9 [7]. Furthermore, Th9 cell proportions have also been observed to be significantly elevated in the peripheral blood of adults with

Correspondence: Yubao Cui, Ph.D, Department of Clinical Laboratory, Wuxi People's Hospital Affiliated to Nanjing Medical University, 299 Qingyang Rd, 214023 Wuxi, China, e-mail: ybcui1975@ hotmail.com Submitted: 29.09.2017; Accepted: 1.08.2018 
allergic asthma, and have been shown to play a key role in allergen-induced airway inflammation $[8,9]$.

Similarly, various cytokines have been demonstrated to be critical for allergic airway inflammation and Th cell differentiation. Atopic infants demonstrate significantly higher levels of IL-9 in the serum than non-atopic infants [10]. With respect to Th cell differentiation, cytokine IL-4 stimulation results in Th2 cell polarization, while transforming growth factor $\beta$ (TGF- $\beta$ ) contributes to inducible regulatory $\mathrm{T}$ cell differentiation [11]. However, the presence of both TGF- $\beta$ and IL-4 initiates CD4 ${ }^{+}$T cell differentiation into Th9 cells [12]. Additionally, IL-1 and IL-33 stimulation has been linked with the induction of Th9 cell differentiation via nuclear factor $\kappa \mathrm{B}$ activation [13, 14]. Despite the detailed understanding of Th9 cells and the related cytokines in adult asthma, very little information is available about their role in recurrent wheezing in infants.

Thus, to better understand the contribution of Th9 cells in the pathogenesis of recurrent wheezing in infants, we assessed the population of Th9 cells in their peripheral blood along with that in healthy age-matched controls. In addition, we measured the plasma concentrations of Th1 cytokines IL-1 $\beta$, IL-6, IL-9, IL-10, IL-17, and IL-33 and TGF- $\beta 1$ as well as the Th2-cytokines IL-4, IL-5, and IL-13. Furthermore, we analyzed the correlation between cytokine levels and the Th9 cell population, with the aim to provide a foundation for future work to better understand immune regulation and the pathogenesis of wheezing in infants.

\section{Material and methods}

\section{Study participants}

The current study was approved (EC/2015/004) by the ethics committee of Binhai Hospital, and written informed consents were obtained from all parents/guardians of the infants. All infants were hospitalized at the Department of Pediatrics of Binhai County Hospital (Yanchen, Jiangsu Province, China) between November 2015 and March 2016.

This case-control study included 34 newly diagnosed infants with recurrent wheezing (age range, 6 to 36 months) and 34 age-matched healthy controls. Inclusion criteria included patients aged between 6 months and 3 -years-old with two or more recurrent hospitalizations due to wheezing. The respiratory symptoms included wheezing and coughing, followed by tachypnea, rales, and the use of accessory muscles. However, infants with pneumonia on immune-suppressing medication, or having any immune system disorder were excluded from this study. Any treatment for wheezing was suspended for 1 month before entering into this study. Importantly, the participants in the control group were negative for atopy, and had no asthma or rhinitis symptoms. There were no statistically significant differences in the ages between the two groups.
The demographic, clinical, and atopic characteristics of the participants are presented in Table 1.

\section{Blood sample preparation}

A $5 \mathrm{ml}$ sample of peripheral blood was collected from each participant in a heparin-coated tube. A $500 \mu \mathrm{l}$ aliquot of each sample was used for the flow cytometric analysis of Th9 cells within $24 \mathrm{~h}$; a $1.5 \mathrm{ml}$ volume was used for the preparation of plasma for cytokine analysis, and the remaining $3 \mathrm{ml}$ volume was used for the isolation of peripheral blood mononuclear cells (PBMCs).

\section{Flow cytometric detection of Th9 cells in the peripheral blood}

For Th9 cell detection, peripheral blood samples $(100 \mu \mathrm{l})$ were activated with phorbol 12-myristate 13-acetate $(50 \mathrm{ng} / \mathrm{ml})$ and ionomycin $(1 \mu \mathrm{g} / \mathrm{ml})$ in the presence of $1 \mu \mathrm{g} / \mathrm{ml}$ brefeldin (Sigma, St. Louis, MO, USA) for $4 \mathrm{~h}$ at $37^{\circ} \mathrm{C}$. Subsequently, the cells were stained with fluorescein isothiocyanate-conjugated anti-CD3 and phycoerythrin (PE)-conjugated anti-CD4 monoclonal antibodies (BD Biosciences, San Jose, CA, USA). After the lysis of red blood cells, they were fixed and permeabilized by adding fixation and permeabilization buffers, respectively (BD Biosciences, San Jose, CA, USA). Next, the cells were labeled with peridinin chlorophyll-Cy5.5-labeled anti-IL-9, APC-anti-IL-4 antibody, and PE-Cy7-labeled anti-IL-17 antibodies (BD Biosciences, San Jose, CA, USA) for $30 \mathrm{~min}$. Finally, the cells were suspended in $200 \mu \mathrm{l}$ of phosphate-buffered saline and immediately analyzed by flow cytometry (Becton Dickinson, San Diego, CA, USA). Among the $\mathrm{CD}^{+}{ }^{+} \mathrm{T}$ cells, the Th9 cell population displayed a phenotype of CD4+ IL-9 ${ }^{+} \mathrm{IL}-4^{-} \mathrm{IL}_{-1} 7^{-}$.

\section{RNA isolation and reverse transcription- polymerase chain reaction analysis}

PBMCs were purified from the blood samples using the Ficoll-Hypaque method (Hengxin, Shanghai, China),

Table 1. Clinical characteristics of wheezing infants and healthy controls

\begin{tabular}{lcc}
\hline Characteristic & Wheezing infants & Controls \\
\hline Age, years & $1.5 \pm 0.6$ & $1.6 \pm 0.6$ \\
\hline Gender, F/M & $10 / 24$ & $11 / 23$ \\
\hline Serum total IgE, ng/ml & $275.4 \pm 97.2^{*}$ & $124.6 \pm 35.2$ \\
\hline Lymphocytes, $\%$ & $62.33 \pm 7.21$ & $63.01 \pm 7.73$ \\
\hline Lymphocytes, $\times 10^{9} / 1$ & $5.27 \pm 1.22$ & $5.18 \pm 1.42$ \\
\hline Eosinophils, $\%$ & $3.16 \pm 0.87^{*}$ & $2.01 \pm 0.68$ \\
\hline Eosinophils, $\times 10^{9} / 1$ & $0.30 \pm 0.13^{*}$ & $0.18 \pm 0.07$ \\
\hline${ }^{*} p<0.05$ for infants with wheezing versus healthy infants &
\end{tabular}


and total RNA was extracted from them using TRIzol reagent (Invitrogen, Carlsbad, CA, USA). cDNA synthesis and amplification were performed using cDNA reverse transcription kit and QuantiTect SYBR Green PCR kit (Invitrogen, Carlsbad, CA, USA), respectively. The following primer sequences were designed: PU.1 sense: 5'-TGAGAAGGACAGGGAGCCAA-3', and antisense: 5'-GAGAAGCTGAGTGCCATGCA-3'; IRF4 sense: 5'-GAAGCCTTGGCGTTCTCA GAC-3', and antisense: 5'-CGTATGTCCATGGGAGATCCG-3'; $\beta$-actin, sense: 5'-TGGCACCCAGCACAATGAA-3', and antisense: 5'-CTAAGTCATA GTCCGCCTAGAAGCA-3'. Reactions were run on a thermal cycler as follows: $1 \mathrm{~min}$ at $95^{\circ} \mathrm{C}$, followed by 40 cycles at $95^{\circ} \mathrm{C}$ for $15 \mathrm{~s}$ and $60^{\circ} \mathrm{C}$ for 1 min. Reverse transcription-polymerase chain reactions (RT-PCR) were performed using the 7500 PCR system (Applied Biosystems, USA). Relative mRNA levels of PU.1 and IRF4 were calculated by the $2^{-\Delta \Delta C t}$ method.

\section{Cytokine analysis}

Plasma separated from the peripheral blood was immediately stored at $-80^{\circ} \mathrm{C}$ until cytokine analysis. The detection of cytokines IL-1 $\beta$, IL-6, IL-10, IL-17A, and IL-33 and TGF- $\beta 1$ was performed following the manufacturer's protocol (Bio-Rad Laboratories, Hercules, CA, USA). Their concentrations were determined using Luminex 200 (Luminex Corporation, Austin, TX, USA) instrument. Each sample was measured twice, and the mean value was used for statistical analysis. The minimum detectable concentrations of IL-1 $\beta$, IL-4, IL-5, IL-6, IL-9, IL-10, IL-13, IL-17A, and IL-33 and TGF- $\beta 1$ in the serum were $3.12 \mathrm{pg} / \mathrm{ml}$, $13.10 \mathrm{pg} / \mathrm{ml}, 4.08 \mathrm{pg} / \mathrm{ml}, 1.34 \mathrm{pg} / \mathrm{ml}, 6.17 \mathrm{pg} / \mathrm{ml}, 2.16 \mathrm{pg} / \mathrm{ml}$, $3.99 \mathrm{pg} / \mathrm{ml}, 9.21 \mathrm{pg} / \mathrm{ml}, 0.68 \mathrm{pg} / \mathrm{ml}$, and $6.12 \mathrm{pg} / \mathrm{ml}$, respectively.

\section{Statistical analysis}

Statistical analysis was performed with Stata 7.0 (StataCorp, College Station, TX, USA) software. The skewed data were expressed as medians $\left(\mathrm{M}, 25^{\text {th }}-75^{\text {th }}\right.$ percentile), and comparison between the two groups was performed using the two-tailed Mann-Whitney U test and Kruskal-Wallis test. The normal distribution of data was presented as the mean \pm standard deviation $(\bar{X} \pm S)$, and the statistical significance between the two groups was compared using the Student's $t$-test. Linear correlations were calculated by Spearman coefficients. The value $p<0.05$ was considered to be statistically significant.

\section{Results}

\section{Wheezing infants displayed an increased Th9 cell population}

First, we analyzed the percentage of Th9 cells in the peripheral blood of a young pediatric population by gating for the $\mathrm{CD}^{+} \mathrm{T}$ cell population (Figs. 1A-1C). Infants with wheezing showed higher percentages of Th9 cells (median, $0.69 \%$; range, $0.46-1.08 \%$ ) as compared to healthy infants (median, 0.25\%; range, 0.13-0.36\%; $p<0.05$; Fig. 1C).

Next, we assessed the levels of PU.1 and IRF4 mRNA expression in PBMCs by RT-PCR. The expression levels of both of these transcription factors were significantly higher in wheezing patients than in healthy infants $(p<0.05$; Fig. 1D). In addition, we observed a positive correlation between the percentage of Th9 cells and PU.1 mRNA $(r=0.449, p<0.05$; Fig. 1E) as well as IRF4 mRNA $(r=0.429, p<0.05$; Fig. 1F) levels in wheezing patients.

\section{Altered levels of circulating Th9- and Th2- related cytokines in wheezing patients}

As cytokines affect Th9 cell differentiation and function, we assessed the plasma concentrations of cytokines IL-1 $\beta$, IL-4, IL-9, IL-17A, and IL-33 and TGF- $\beta 1$. Higher levels of IL-4, IL-9, and IL-33 and TGF- $\beta 1$ were observed in wheezing infants, compared to healthy controls $(p<0.05$; Figs. 2A-1D). However, there were no significant differences in the IL-1 $\beta$ and IL-17A levels between the two groups ( $p>0.05$; Figs. 2E and 2F). Adults with asthma are typically characterized by Th2 cytokines, such as IL-4, IL-5, and IL-13 [15]; however, our analysis did not show any difference in the IL-5 and IL-13 levels between the wheezing and control groups $(p>0.05$; Figs. 3A and $3 \mathrm{~B}$ ). Moreover, as wheezing is caused by airway inflammation, we also measured the levels of the proinflammatory cytokine IL-6 and the anti-inflammatory cytokine IL-10. Surprisingly, we observed that wheezing infants had significantly higher levels of IL-10 and lower levels of IL-6 as compared to healthy controls $(p<0.05$; Figs. 3C and 3D).

\section{Correlation between Th9 cells and plasma levels of cytokines IL-4 and IL-9 in wheezing patients}

To further explore the possible role of cytokines in Th9 cell differentiation, we investigated the correlation between the percentage of Th9 cells and the concentrations of cytokines IL-4, IL-6, IL-9, IL-10, and IL-33 and TGF- $\beta 1$ in the plasma of wheezing patients. We detected a positive correlation between the percentage of Th9 cells and the concentrations of IL-9 ( $r=0.644, p<0.05$; Fig. 4A) and IL-4 ( $r=0.408, p<0.05$; Fig. 4B) in the plasma. However, we did not observe any correlation of Th9 cells with the concentrations of IL-6, IL-10, or IL-33, or TGF- $\beta 1$ $(p>0.05$; Figs. 4C-4F).

\section{Discussion}

This study revealed several novel findings. First, the percentage of Th9 cells was significantly higher in 

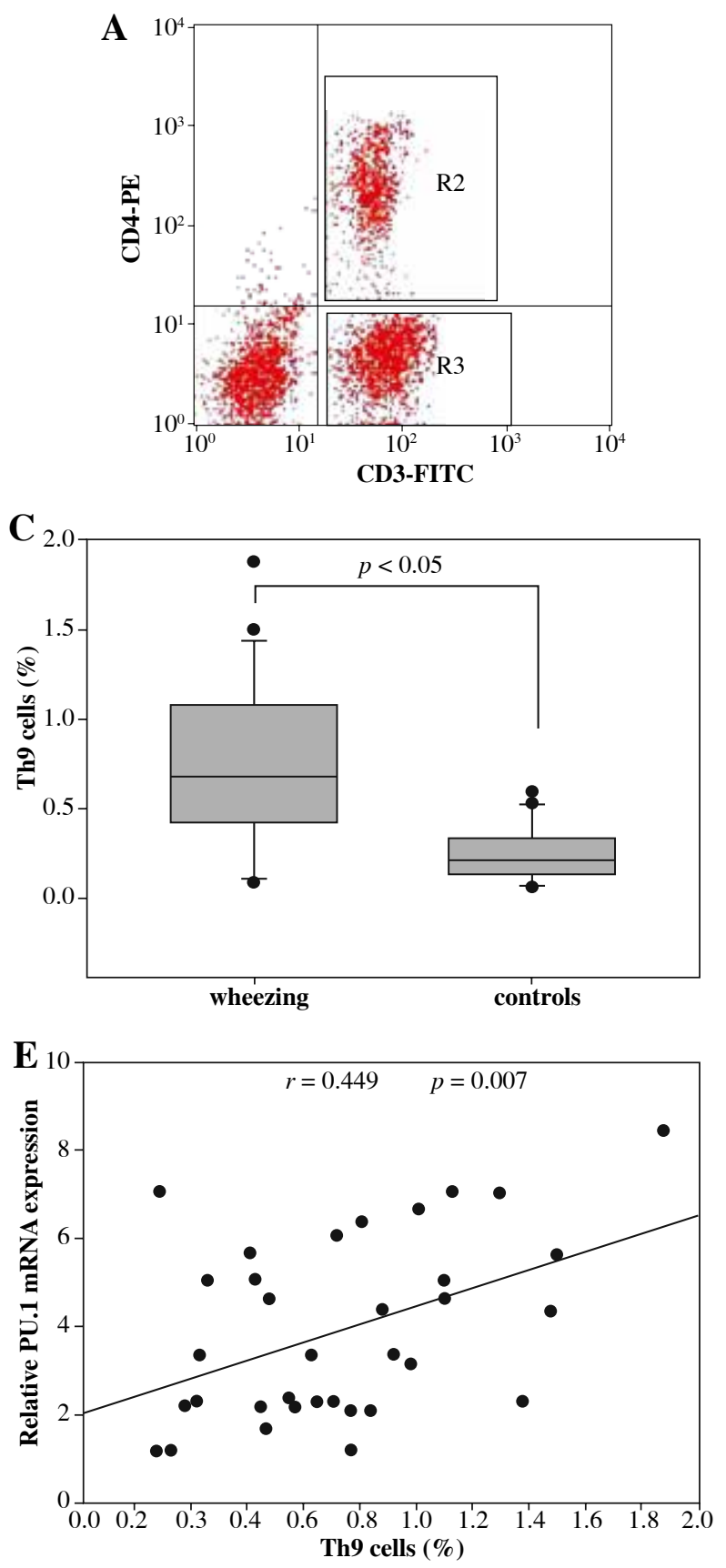
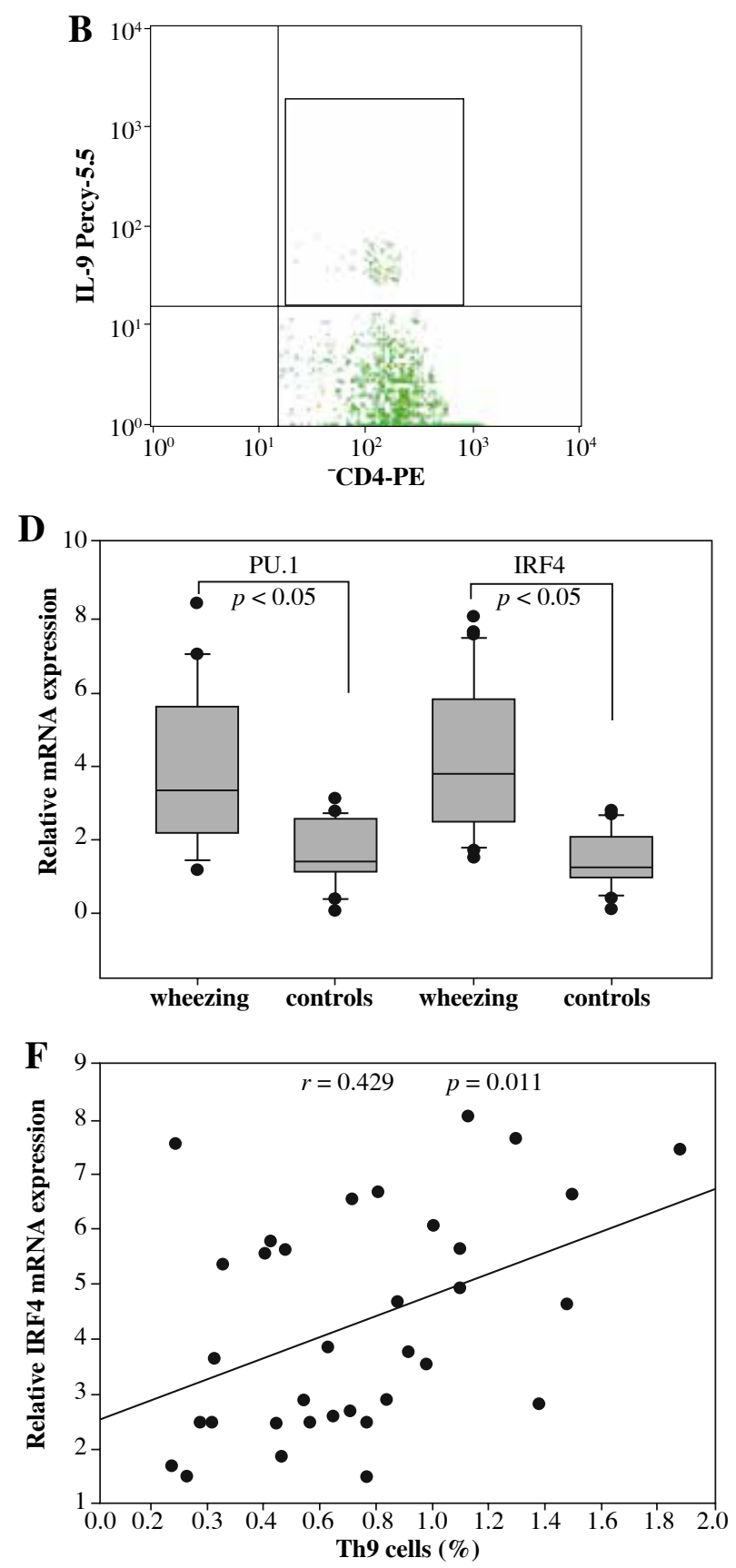

Fig. 1. Analysis of the Th9 cell population. Peripheral blood samples from wheezing infants and controls were stained and then analyzed by flow cytometry. (A) Plot showing CD4 ${ }^{+}$T cells on gated CD $3^{+}$lymphocytes. (B) Plot showing IL-9 expression on gated $\mathrm{CD}^{+} \mathrm{CD}^{+}$lymphocytes. $(\mathbf{C})$ Percentages of $\mathrm{Th} 9$ cells among $\mathrm{CD} 4^{+} \mathrm{T}$ cells from healthy controls and wheezing infants. (D) RT-PCR analysis of PU.1 and IRF4 mRNA expression in PBMCs from wheezing infants and healthy controls. (E) Correlation analysis between the percentage of Th9 cells and the PU.1 mRNA expression in PBMCs from wheezing patients. (F) Correlation analysis between the percentage of Th9 cells and the IRF4 mRNA expression in PBMCs from wheezing patients. Box plots represent the median $\left(25^{\text {th }}-75^{\text {th }}\right.$ percentile $)$, and dots represent outliers. Statistical significance was determined by the Kruskal-Wallis test. $r$ denotes Spearman's correlation coefficient, and a $p$ value less than 0.05 represents statistical significance 

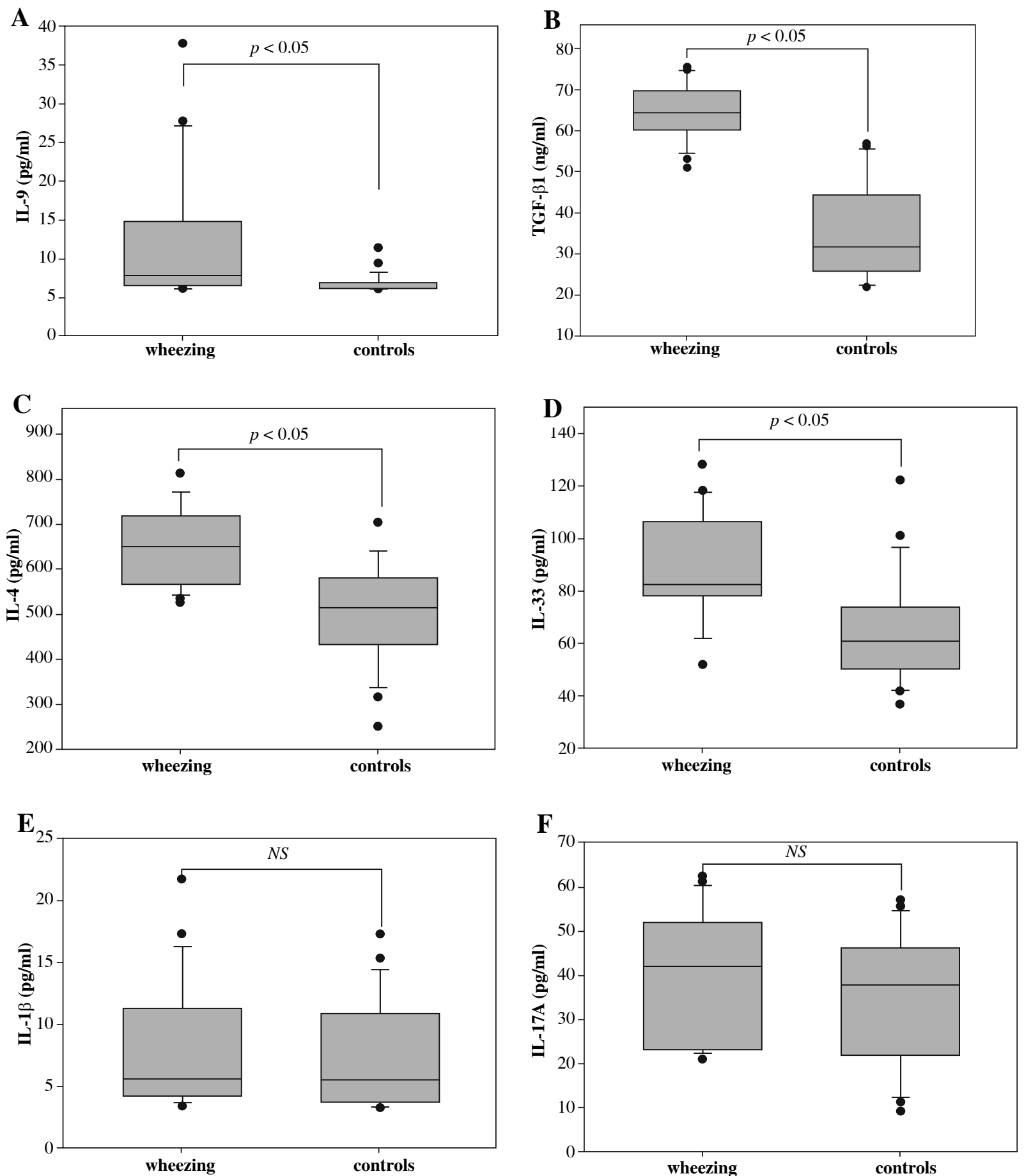

Fig. 2. Comparative analysis of Th9-related cytokines in wheezing infants and control group. Plasma levels of the Th9-related cytokines IL-9, TGF- $\beta 1$, IL-4, IL-33, IL-1 $\beta$, and IL-17A were analyzed by a Luminex 200 immunoassay. Box plots represent the median (25th-75th percentile), and dots represent outliers. Statistical significance was determined by the Kruskal-Wallis test, and $p<0.05$ represents statistical significance

the peripheral blood of infants with recurrent wheezing, compared to healthy controls. In addition, we observed a positive correlation of Th9 cells with PU.1 mRNA and
IL-9 levels in wheezing infants. These observations were consistent because Th9 cells have been shown to regulate IL-9 production through the transcription factor PU.1. 
A

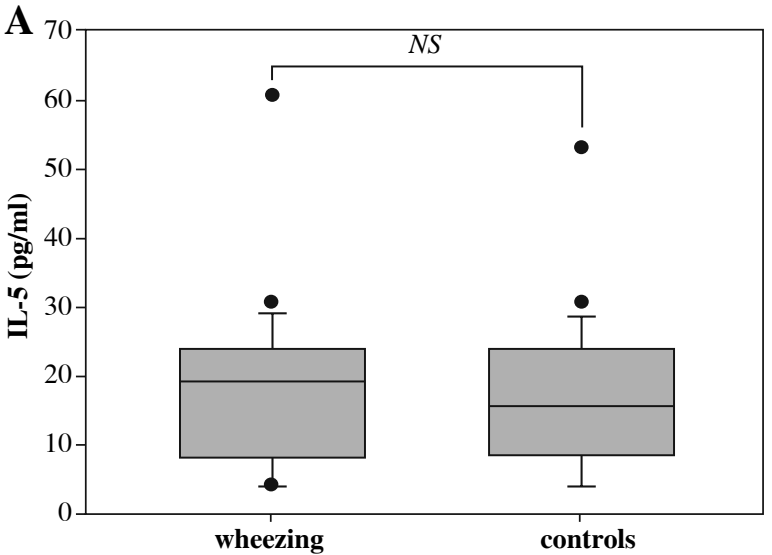

$\mathbf{C}_{350}$

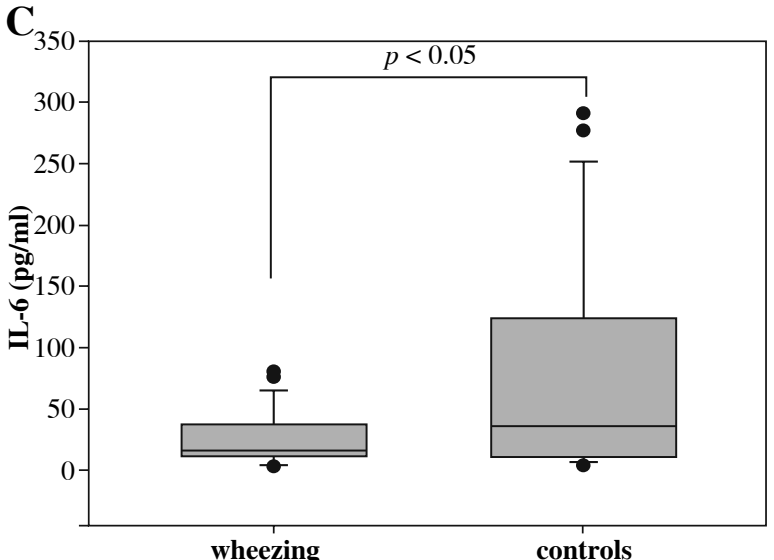

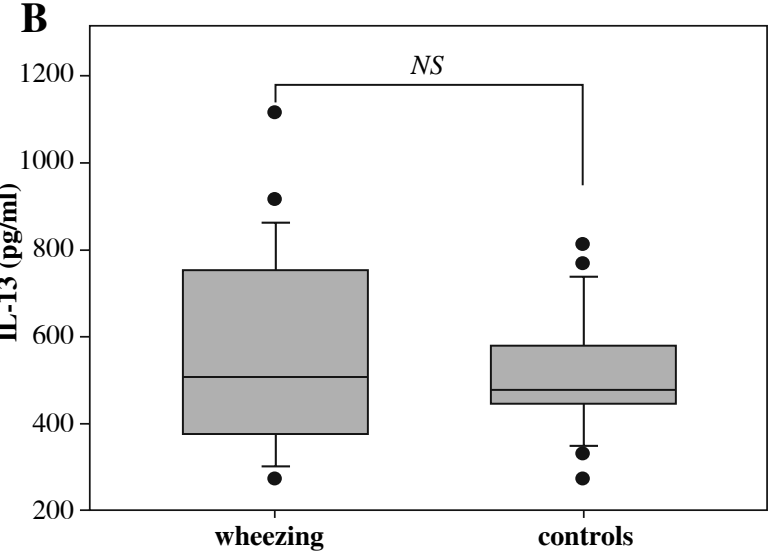

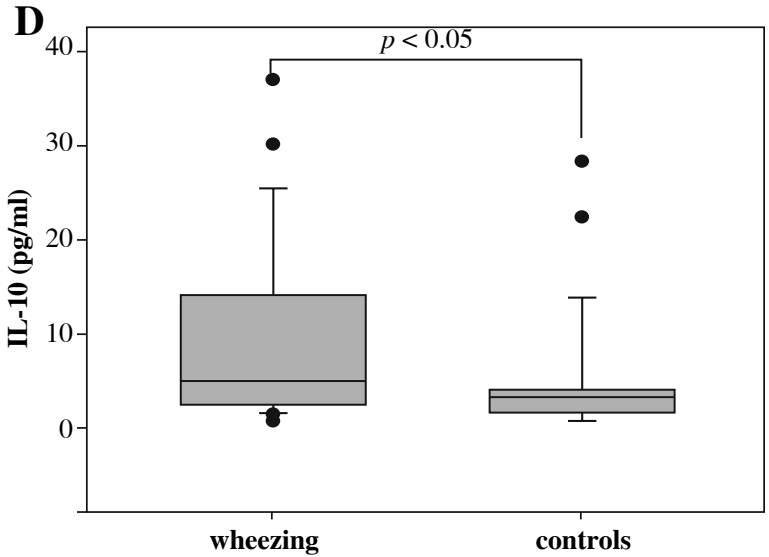

Fig. 3. Comparative analysis of Th2-related cytokines in wheezing infants and control group. Plasma levels of the Th2-related cytokines IL-5 and IL-13, the inflammatory mediator IL-6, and the anti-inflammatory cytokine IL-10 were analyzed by a Luminex 200 immunoassay. Box plots represent the median $\left(25^{\text {th }}-75^{\text {th }}\right.$ percentile), and dots represent outliers. Statistical significance was determined by the Kruskal-Wallis test, and $p<0.05$ represents statistical significance

Second, infants with recurrent wheezing exhibited higher circulating levels of Th9-related cytokines such as IL-4, and IL-33; TGF- $\beta 1$ and these cytokines play an important role in Th9 cell differentiation. Finally, we also observed surprisingly lower levels of the proinflammatory cytokine IL-6 and higher levels of the anti-inflammatory cytokine IL-10 in the wheezing infants.

Several studies have demonstrated the association between an increased risk of early persistent wheezing and allergic sensitization [16, 17]. Our data revealed that wheezing infants had increased proportions of Th9 cells as well as higher levels of PU.1 and IRF4 mRNA along with IL-9 protein in the peripheral blood, compared to healthy infants. These results indicated that Th9 cells are also altered in wheezing infants, just like in adults with allergic asthma [8, 9]. Although IL-9 has been shown to be produced by several other cell types, including type 2 cytokine-producing innate lymphoid cells, eosinophils, and mast cells [18], in our study, we found a positive cor- relation of IL-9 production with Th9 cell percentages. We therefore speculate that, in wheezing infants, Th9 cells are the main source of IL-9.

Additionally, an association between plasma TGF- $\beta 1$ and IL-4 levels, and the risk of asthma in children has also been reported $[19,20]$. Consistent with this finding, we also noticed significantly higher levels of TGF- $\beta 1$ and IL-4 in the plasma of infants in the wheezing group, compared to the healthy group. These cytokines may account for the increased Th9 differentiation. In addition, IL-33 has been implicated in Th9 cell differentiation as well as in exacerbating asthma in children $[13,21]$. Our data confirmed this report, as we also observed higher concentration of IL-33 in infants with wheezing. Interestingly, IL-33 is reported to be a corticosteroid-resistant mediator of severe asthma in children [22]. On the other hand, IL-1 $\beta$ has been shown to induce Th9 differentiation [14]; however, in our study, we did not see any difference in IL- $1 \beta$ levels between infants with wheezing and controls. 

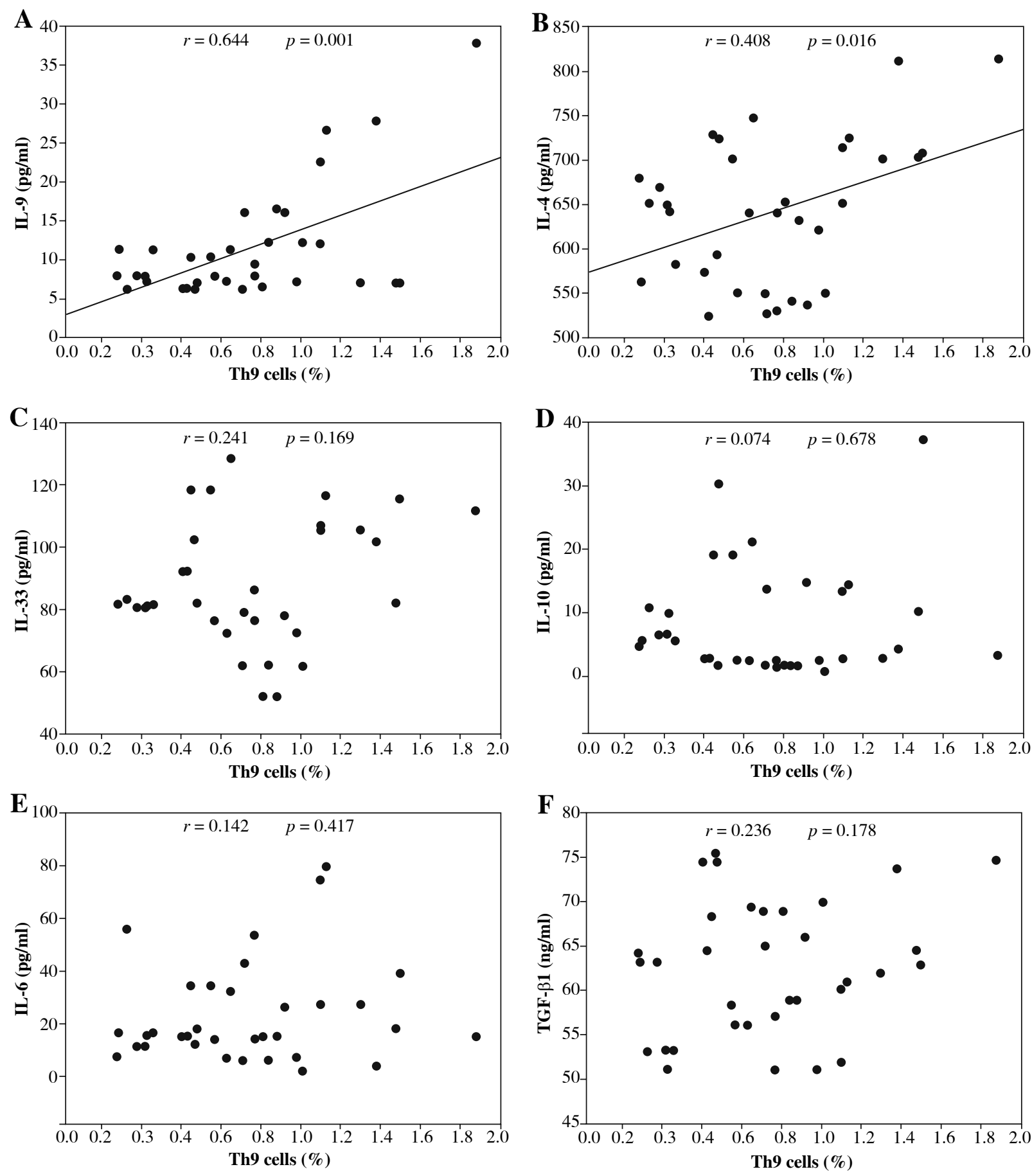

Fig. 4. Relationship between plasma cytokines and Th9 cells in wheezing infants. Panels A-F show the correlation between Th9 cells and the plasma levels of IL-9, IL-4, IL-33, IL-10, IL-6, and TGF- $\beta 1$ in infants with wheezing. $r$ denotes Spearman's correlation coefficient, and a $p$ value less than 0.05 represents statistical significance

IL-9, through induction of the JAK1 pathway, can also stimulate the proliferation of Th17 cells [23], and Th17 cytokines such as IL-17A-F are believed to be critical for airway hyper-responsiveness [24]. However, in our study, the levels of IL-17 were similar between wheezing and healthy infants. One tentative explanation could be the presence of lower levels of IL-6 in wheezing infants, which happens to be a key cytokine for Th17 cell differentiation. In addition, we observed higher levels of the anti-inflammatory factor IL-10 in wheezing infants, which is consistent with previ- 
ously published reports $[25,26]$. The study by Burgess et al. has shown that elevated serum levels of IL-10 in early life predict increased numbers of wheezing episodes [26]. However, the reason for the lower levels of IL- 6 and the higher levels of IL-10 in wheezing infants is not very clear; it has been speculated that asthma in preschool children are often associated with respiratory virus infections, which may result in a higher level of IL-10 during disease onset [27, 28]. An alternative explanation is based on the "hygiene hypothesis," which suggests that the absence of infections in early life may lead to an increased risk of developing asthma [29].

In addition, Th2 cell cytokines such as IL-4, IL-5, and IL-13 have been shown to play key roles in adult asthma [29]. IL-4 influences Th2 cell polarization, while IL-5 is predominantly involved in eosinophil differentiation and function. Furthermore, IL-13 is critical for mucous secretion and airway hyper-responsiveness [29]. Our study observed similar levels of IL-5 and IL-13 between wheezing infants and controls. The dominance of the Th2 profile in children with asthma has been controversial as therapy targeting IL-5 has been demonstrated to be very effective in adults only, while its effectiveness is uncertain in children with asthma [30]. Moreover, the serum levels of IL-5 have been shown to be significantly correlated with age and were lower in acute asthma patients younger than 3-years-old than among those older than 3-years-old [31]. Thus, Th9 cells may have a greater functional role in infants with recurrent wheezing.

However, there are several limitations in this study. First, the sample sizes of infants with recurrent wheezing and healthy controls were relatively small. Second, recurrent wheezing is also related to environmental exposures, but our samples were primarily collected in the winter season [4]. It is presumed that allergen exposure, pollution, and infection have different effects on infants with wheezing at different ages and are accompanied by different clinical phenotypes [3]. Thus, in future studies, we intend to investigate the effect of Th9 cells underlying each of these precipitating factors.

\section{Conclusions}

Our study demonstrated that the percentage of Th9 cells in the peripheral blood of infants with recurrent wheezing was significantly higher than that in healthy controls. Furthermore, infants with wheezing exhibited higher levels of the circulating Th9-related cytokines TGF- $\beta 1$, IL-4, and IL-33. Overall, our data indicate that a larger proportion of Th9 cells may play a role in the pathogenesis of recurrent wheezing in infants.

\section{Acknowledgments}

This work was supported by a project of the Medical and Health Technology Development Program in Yancheng City, China (YK2015065).
The authors declare no conflict of interest.

\section{References}

1. Cook J, Saglani S (2016): Pathogenesis and prevention strategies of severe asthma exacerbations in children. Curr Opin Pulm Med 22: 25-31.

2. Landgraf-Rauf K, Anselm B, Schaub B (2016): The puzzle of immune phenotypes of childhood asthma. Mol Cell Pediatr 3: 27.

3. Fogaca HR, Marson FA, Toro AA, et al. (2014): Epidemiological aspects of and risk factors for wheezing in the first year of life. J Bras Pneumol 40: 617-625.

4. Patelarou E, Tzanakis N, Kelly FJ (2015): Exposure to indoor pollutants and Wheeze and asthma development during early childhood. Int J Environ Res Public Health 12: 3993-4017.

5. Jabeen R, Goswami R, Awe O, et al. (2013): Th9 cell development requires a BATF regulated transcriptional network. J Clin Invest 123: 4641-4653.

6. Yao W, Tepper RS, Kaplan MH (2011): Predisposition to the development of IL-9-secreting T cells in atopic infants. J Allergy Clin Immunol 128: 1357-1360.

7. Brough HA, Cousins DJ, Munteanu A, et al. (2014): IL-9 is a key component of memory TH cell peanut-specific responses from children with peanut allergy. J Allergy Clin Immunol 134: 1329-1338.

8. Hoppenot D, Malakauskas K, Lavinskien S, et al. (2015): Peripheral blood Th9 cells and eosinophil apoptosis in asthma patients. Medicina (Kaunas) 51: 10-17.

9. Hoppenot D, Malakauskas K, Lavinskiene S, et al. (2015): p-STAT6, PU.1, and NF-kB are involved in allergen-induced late-phase airway inflammation in asthma patients. BMC Pulm Med 15: 122.

10. Yao W, Zhang Y, Jabeen R, et al. (2013): Interleukin-9 is required for allergic airway inflammation mediated by the cytokine TSLP. Immunity 38: 360-372.

11. Caza T, Landas S (2015): Functional and phenotypic plasticity of CD4+ T cell subsets. Biomed Res Int 2015: 1-13.

12. Veldhoen M, Uyttenhove C, Van Snick JA, et al. (2008): Transforming growth factor-beta reprograms the differentiation of $\mathrm{T}$ helper 2 cells and promotes an interleukin 9-producing subset. Nat Immunol 9: 1341-1346.

13. Blom L, Poulsen BC, Jensen BM, et al. (2011): IL-33 induces IL-9 production in human CD4+ T cells and basophils. PLoS One 6: e21695.

14. Ye ZJ, Yuan ML, Zhou Q, et al. (2012): Differentiation and recruitment of Th9 cells stimulated by pleural mesothelial cells in human Mycobacterium tuberculosis infection. PLoS One 7: e31710.

15. Fahy JV (2015): Type 2 inflammation in asthma-present in most, absent in many. Nat Rev Immunol 15: 57-65.

16. Rubner FJ, Jackson DJ, Evans MD, et al. (2017): Early life rhinovirus wheezing, allergic sensitization, and asthma risk at adolescence. J Allergy Clin Immunol 139: 501.

17. Melen E, Kere J, Pershagen G, et al. (2004): Influence of male sex and parental allergic disease on childhood wheezing: role of interactions. Clin Exp Allergy 34: 839-844.

18. Mohapatra A, Van Dyken SJ, Schneider C (2016): Group 2 innate lymphoid cells utilize the IRF4-IL-9 module to coordinate epithelial cell maintenance of lung homeostasis. Mucosal Immunol 9: 275-286. 
19. Tsai TC, Lu JH, Chen SJ, Tang RB (2009): Soluble interleukin-10 and transforming growth factor-beta in children with acute exacerbation of allergic asthma. J Asthma 46: 21-24.

20. Al-Daghri NM, Alokail MS, Draz HM, et al. (2014): Th1/ Th2 cytokine pattern in Arab children with severe asthma. Int J Clin Exp Med 7: 2286-2291.

21. Bahrami Mahneh S, Movahedi M, Aryan Z, et al. (2015): Serum IL-33 Is Elevated in Children with Asthma and Is Associated with Disease Severity. Int Arch Allergy Immunol 168: 193-196.

22. Saglani S, Lui S, Ullmann N, et al. (2013): IL-33 promotes airway remodeling in pediatric patients with severe steroid-resistant asthma. J Allergy Clin Immunol 132: 676-685.

23. Elyaman W, Bradshaw EM, Uyttenhove C, et al. (2009): IL-9 induces differentiation of TH17 cells and enhances function of FoxP3+natural regulatory T cells. Proc Natl Acad Sci USA 106: 12885-12890.

24. Apostolova P, Zeiser R (2016): The role of purine metabolites as DAMPs in acute graft-versus-host disease. Front Immunol 7: 439.

25. Yu ZW, Qian J, Gu XH, et al. (2015): Changes in serum inflammatory factors in wheezing infants with community-acquired pneumonia. China J Conte Ped 17: 815-818.

26. Burgess SL, Lu M, Ma JZ, et al. (2016): Inflammatory markers predict episodes of wheezing during the first year of life in Bangladesh. Respir Med 110: 53-57.

27. Grissell TV, Powell H, Shafren DR, et al. (2005): Interleukin-10 gene expression in acute virus-induced asthma. Am J Respir M172: 433-439.

28. Song W, Yu Z, Doran SF, et al. (2015): Respiratory syncytial virus infection increases chlorine-induced airway hyperresponsiveness. Am J Physiol Lung Cell Mol Physiol 309: L205-210.

29. Hansbro PM, Starkey MR, Mattes J, Horvat JC (2014): Pulmonary Immunity during Respiratory Infections in Early Life and the Development of Severe Asthma. Ann Am Thorac Soc 11: 297-302.

30. Dunican EM, Fahy JV (2015): The Role of Type 2 Inflammation in the Pathogenesis of Asthma Exacerbations. Ann Am Thorac Soc 12: 144-149.

31. He XY, Simpson JL, Wang F (2011): Inflammatory phenotypes in stable and acute childhood asthma. Paediatr Respir Rev 12: 165-169.

32. Kato M, Suzuki K, Yamada Y, et al. (2015): Virus detection and cytokine profile in relation to age among acute exacerbations of childhood asthma. Allergol Int 64: 64-70. 